\title{
Alternative Exposure Parameters and Post Process Noise Reduction Expect Considerable Dose Reduction in Single Mammography Views-Initial Experience on Mastectomy Specimens
}

\author{
Adriana M. J. Bluekens, ${ }^{1,2 *}$, Ruben Evan Engen ${ }^{2}$, Nico Karssemeijer ${ }^{3}$, Klaas H. Schuur ${ }^{1,2}$, \\ Mireille J. M. Broeders ${ }^{2,4}$, Gerard J. den Heeten ${ }^{2,5}$ \\ ${ }^{1}$ St. Elisabeth Hospital, Department of Radiology, Tilburg, The Netherlands \\ ${ }^{2}$ National Expert and Training Centre for Breast Cancer Screening, Nijmegen, The Netherlands \\ ${ }^{3}$ Department of Radiology, Radboud University Nijmegen Medical Centre, Nijmegen, The Netherlands \\ ${ }^{4}$ Department for Health Evidence, Radboud University Nijmegen Medical Centre, Nijmegen, The Netherlands \\ ${ }^{5}$ Department of Radiology, Academic Medical Centre (AMC), Amsterdam, The Netherlands \\ Email: ${ }^{*}$ n.bluekens@elisabeth.nl
}

Received May 23, 2013; revised June 21, 2013; accepted June 28, 2013

Copyright (C) 2013 Adriana M. J. Bluekens et al. This is an open access article distributed under the Creative Commons Attribution License, which permits unrestricted use, distribution, and reproduction in any medium, provided the original work is properly cited.

\begin{abstract}
Objective: To experimentally indicate a lower limit of dose in mammographic imaging yielding adequate image quality for complementary diagnostic views, by evaluation of image series with different exposure parameters and additional image processing on mastectomy specimens with diverse pathology. Methods: Image series were obtained on seven specimens with different target-filter combinations at different exposure values. Three experienced radiologists assessed the lowest acceptable dose level per specimen using a relative grading technique. With the standard image as reference, fibroglandular tissue and pathological structures, including microcalcifications, were evaluated. Subsequently, a series of pixel binning processes was tested and subjectively assessed on the selected images. Results: The lowest dose level at which image quality was acceptable, and achieved with a W/Ag target-filter combination at $32 \mathrm{kV}$ and $4 \mathrm{mAs}$. These images can be acquired with $10.4 \%$ to $22.4 \%$ of the average glandular dose in standard images. Post process pixel binning added to the interpretability of such low dose images. Conclusion: This specimen study suggests that dose level of mammography images might be reduced substantially by general application of a W/Ag spectrum, particularly when combined with post process noise reduction. Future studies should focus on the feasibility of this technique in clinical mammography.
\end{abstract}

Keywords: Digital Mammography; Dose Reduction

\section{Introduction}

Mammography is one of the most widely used diagnostic modalities, playing a major role in the detection of early breast cancer. The ever increasing demand for mammography, in a largely asymptomatic population, calls for optimisation of radiation dose in mammography.

In digital mammography the image acquisition and processing can be customised, as exposure parameters and post processing algorithms can be adjusted separately. As a result, digital mammography has the potential to obtain and optimise images with a low radiation dose

"Corresponding author. level for specific purposes. Dosimetry studies carried out for digital mammography [1-3], suggest a possible 50\% dose reduction, as the radiologists' performance in standard and dose reduced images did not differ significantly. All of these were experimental and performed with phantoms or manipulated mammography images. Although the effects of dose reduction on image quality parameters are well known, the balance between dose and perceived image quality is still unclear.

The current digital mammography units are quantum noise limited for a very wide range of dose levels, including (very) low dose levels. With low signal and relatively high noise level the quality of low dose images is restric- 
ted, due to a relatively low number of X-ray quanta, resulting in high quantum noise, in which the signal is difficult to detect. The use of a tungsten/silver (W/Ag) target-filter combination increases the number of X-ray quanta reaching the detector, while the absorbed dose for the breast is similar. This reduces the amount of quantum noise and therefore increases the visibility of structures. Therefore, the use of a W/Ag spectrum might be particularly interesting in dose reduced imaging. The expected noise level in (very) low dose images might be decreased further by post process noise reduction techniques. There are previous studies that show potential for dose reduction with different beam qualities [4,5], but W/Ag images were not included in the evaluation.

Most studies focus on generalised dose reduction in mammography, with consequent concerns about the potential loss of image quality and the resultant effect on diagnostic efficacy [1-3]. The aim of this pilot study is to investigate whether a differentiated approach could be feasible, such as dose reduction in specific views only. For that, we were looking for a threshold radiation dose applicable for single views that can add to mammography.

To explore the potential of low dose images we evaluated series of mastectomy specimen images with different exposure parameters and noise reduction settings. Because physical parameters poorly correlate with diagnostic efficacy [6], we choose subjective rating to assess image quality. By relative grading of perceived image quality an indication for a threshold level of radiation dose needed for acquiring mammographic images useful for diagnostics was determined.

\section{Methods}

\subsection{Specimens}

From January to April 2010 we collected whole breast specimens of every mastectomy performed in the St Elisabeth hospital, Tilburg, the Netherlands. In this period we obtained seven specimens with diverse pathology; two stellate lesions, one mass with microcalcifications, and two clusters of microcalcifications. One specimen also contained a well-defined benign mass. Two specimens did not have any visible pathology.

\subsection{Image Acquisition}

All images were acquired on a Lorad Selenia FFDM system (Hologic Inc. Danbury, CT) with $70-\mu$ m pixel size and $24 \times 29 \mathrm{~cm}$ field of view. Dose series of all specimen started with a standard mammography image acquired under automatic exposure control (AEC) conditions. For every specimen the same X-ray spectrum as selected by the AEC, target-filter combination (i.e. tungsten/rhodium $(\mathrm{W} / \mathrm{Rh})$ ) and tube voltage $(\mathrm{kV})$, was used for the image series with incremental exposure values (mAs). These series contained images obtained with circa 5, 10, 15 and $20 \%$ of the mAs-value used with the AEC. For specimens with microcalcifications series were extended up until a $50 \%$ dose level. In case of visible pathology an additional image was acquired with $32 \mathrm{kV}$ tube voltage and the $\mathrm{W} /$ Ag target-filter combination with an exposure comparable to $5 \%$ of the AEC mAs-value. The fractions mentioned are approximations, as mAs-value selection was limited by a minimum value of 3.8 and an interval scale. Incident air kerma (ESAK) values were measured. In addition, the average glandular dose was estimated based on a breast thickness of $4 \mathrm{~cm}$ (Table 1), in accordance with the average specimen compression thickness.

\subsection{Observer Study}

Three screening radiologists, all experienced with digital mammography images, were involved in reading the low dose series. Review was performed independently on a dedicated mammography workstation (SecurView DX, Ho-logic Inc., Danbury, CT) with customised software (MeVis Medical Solutions, Bremen, Germany) and two high-resolution 5-megapixel portrait monitors (Barco NV, Kortrijk, Belgium). Overall perceived image quality and appearance of suspicious lesions were assessed. The quality of the images was rated with the AEC dose image visible on the left screen, while displaying incremental low dose images on the right screen. By instant comparison with the AEC dose image the (loss of) information in the low-dose image could be determined. This sort of relative grading of image quality was applied for three feature categories: fibroglandular tissue, mass (when present), and microcalcifications (when present). Per specimen the diagnostic quality of the images was scored as either acceptable or unacceptable in the presentation of these features. Additionally, the quality of the W/Ag image was indicated by a comparison with the first acceptable image of the Rhseries, using a nominal scoring scale (better/similar/ worse).

\subsection{Image Processing}

The images acquired with parameters that appeared to be most suitable for low dose imaging, as resulted from the observer study, underwent further processing. In an attempt to increase the perceptual image quality post process noise reduction was applied. Hereto, we used a simple method, known as pixel binning, in which the signal in blocks of neighbouring pixels is combined (Figure 1). At the expense of detail information, binning can average out uncorrelated noise effectively, thereby increasing the visibility of all correlated elements in an image like breast structures. As (very) low dose images should not be used for detail analysis the associated decrease in signal transfer at high frequencies is assumed to be less relevant. Binning was done manually, using image analysis soft 
Table 1. Exposure parameters of dose series images per specimen.

\begin{tabular}{|c|c|c|c|c|c|c|}
\hline Specimen & Target & Filter & $\begin{array}{c}\text { Tube } \\
\text { voltage } \\
(\mathbf{k V )}\end{array}$ & $\begin{array}{c}\text { Tube } \\
\text { current } \\
\text { (mAs) }\end{array}$ & $\begin{array}{l}\text { ESAK } \\
\text { (mGy) }\end{array}$ & $\begin{array}{c}\text { AGD } \\
\text { (mGy) }\end{array}$ \\
\hline \multirow[t]{5}{*}{ A } & Tungsten & Rhodium & 26 & $67.2(100 \%)$ & 2.23 & 0.71 \\
\hline & Tungsten & Rhodium & 26 & $3.8(5.7 \%)$ & 0.13 & 0.04 \\
\hline & Tungsten & Rhodium & 26 & $6.7(10.0 \%)$ & 0.22 & 0.07 \\
\hline & Tungsten & Rhodium & 26 & $9.8(14.6 \%)$ & 0.33 & 0.10 \\
\hline & Tungsten & Rhodium & 26 & $13.6(20.2 \%)$ & 0.45 & 0.14 \\
\hline \multirow[t]{5}{*}{ B } & Tungsten & Rhodium & 26 & $71.3(100 \%)$ & 2.37 & 0.75 \\
\hline & Tungsten & Rhodium & 26 & $3.8(5.3 \%)$ & 0.13 & 0.04 \\
\hline & Tungsten & Rhodium & 26 & $6.7(9.4 \%)$ & 0.22 & 0.07 \\
\hline & Tungsten & Rhodium & 26 & $9.8(13.7 \%)$ & 0.33 & 0.10 \\
\hline & Tungsten & Rhodium & 26 & $13.6(19.1 \%)$ & 0.45 & 0.14 \\
\hline \multirow[t]{6}{*}{$\mathrm{C}$} & Tungsten & Rhodium & 26 & $63.4(100 \%)$ & 2.10 & 0.67 \\
\hline & Tungsten & Rhodium & 26 & $3.8(6.0 \%)$ & 0.13 & 0.04 \\
\hline & Tungsten & Rhodium & 26 & $5.7(9.0 \%)$ & 0.19 & 0.06 \\
\hline & Tungsten & Rhodium & 26 & $9.8(15.5 \%)$ & 0.33 & 0.10 \\
\hline & Tungsten & Rhodium & 26 & $11.9(18.8 \%)$ & 0.40 & 0.13 \\
\hline & Tungsten & Silver & 32 & $3.8(6.0 \%)$ & $\begin{array}{c}0.28 \\
(13.3 \%)\end{array}$ & $\begin{array}{c}0.11 \\
(16.4 \%)\end{array}$ \\
\hline \multirow[t]{9}{*}{$\mathrm{D}$} & Tungsten & Rhodium & 26 & $79.5(100 \%)$ & 2.64 & 0.86 \\
\hline & Tungsten & Rhodium & 26 & $3.8(4.8 \%)$ & 0.13 & 0.04 \\
\hline & Tungsten & Rhodium & 26 & $7.8(9.8 \%)$ & 0.26 & 0.08 \\
\hline & Tungsten & Rhodium & 26 & $11.9(15.0 \%)$ & 0.40 & 0.13 \\
\hline & Tungsten & Rhodium & 26 & $15.7(19.7 \%)$ & 0.52 & 0.17 \\
\hline & Tungsten & Rhodium & 26 & $19.6(24.7 \%)$ & 0.65 & 0.21 \\
\hline & Tungsten & Rhodium & 26 & $23.9(30.1 \%)$ & 0.79 & 0.26 \\
\hline & Tungsten & Rhodium & 26 & $40.0(50.3 \%)$ & 1.33 & 0.43 \\
\hline & Tungsten & Silver & 32 & $3.8(4.8 \%)$ & $\begin{array}{c}0.28 \\
(10.6 \%)\end{array}$ & $\begin{array}{c}0.11 \\
(12.8 \%)\end{array}$ \\
\hline \multirow[t]{6}{*}{$\mathrm{E}$} & Tungsten & Rhodium & 27 & $85.3(100 \%)$ & 3.22 & 1.06 \\
\hline & Tungsten & Rhodium & 27 & $3.8(4.5 \%)$ & 0.14 & 0.05 \\
\hline & Tungsten & Rhodium & 27 & $7.8(9.1 \%)$ & 0.29 & 0.10 \\
\hline & Tungsten & Rhodium & 27 & $13.6(15.9 \%)$ & 0.51 & 0.17 \\
\hline & Tungsten & Rhodium & 27 & $17.8(20.9 \%)$ & 0.67 & 0.22 \\
\hline & Tungsten & Silver & 32 & $3.8(4.5 \%)$ & $\begin{array}{c}0.28 \\
(8.7 \%)\end{array}$ & $\begin{array}{c}0.11 \\
(10.4 \%)\end{array}$ \\
\hline \multirow[t]{9}{*}{$\mathrm{F}$} & Tungsten & Rhodium & 25 & $55.1(100 \%)$ & 1.58 & 0.49 \\
\hline & Tungsten & Rhodium & 25 & $3.8(6.9 \%)$ & 0.11 & 0.03 \\
\hline & Tungsten & Rhodium & 25 & $5.7(10.3 \%)$ & 0.16 & 0.05 \\
\hline & Tungsten & Rhodium & 25 & $7.8(14.2 \%)$ & 0.22 & 0.07 \\
\hline & Tungsten & Rhodium & 25 & $11.9(21.6 \%)$ & 0.34 & 0.11 \\
\hline & Tungsten & Rhodium & 25 & $15.7(28.5 \%)$ & 0.45 & 0.14 \\
\hline & Tungsten & Rhodium & 25 & $21.7(39.4 \%)$ & 0.62 & 0.19 \\
\hline & Tungsten & Rhodium & 25 & $27.5(49.9 \%)$ & 0.79 & 0.25 \\
\hline & Tungsten & Silver & 32 & $3.8(6.9 \%)$ & $\begin{array}{c}0.28 \\
(17.7 \%)\end{array}$ & $\begin{array}{c}0.11 \\
(22.4 \%)\end{array}$ \\
\hline \multirow[t]{6}{*}{ G } & Tungsten & Rhodium & 25 & $75.5(100 \%)$ & 2.16 & 0.68 \\
\hline & Tungsten & Rhodium & 25 & $3.8(5.0 \%)$ & 0.11 & 0.03 \\
\hline & Tungsten & Rhodium & 25 & $6.7(8.9 \%)$ & 0.19 & 0.06 \\
\hline & Tungsten & Rhodium & 25 & $11.9(15.8 \%)$ & 0.34 & 0.11 \\
\hline & Tungsten & Rhodium & 25 & $15.7(20.8 \%)$ & 0.45 & 0.14 \\
\hline & Tungsten & Silver & 32 & $3.8(5.0 \%)$ & $\begin{array}{c}0.28 \\
(13.0 \%)\end{array}$ & $\begin{array}{c}0.11 \\
(16.2 \%)\end{array}$ \\
\hline
\end{tabular}

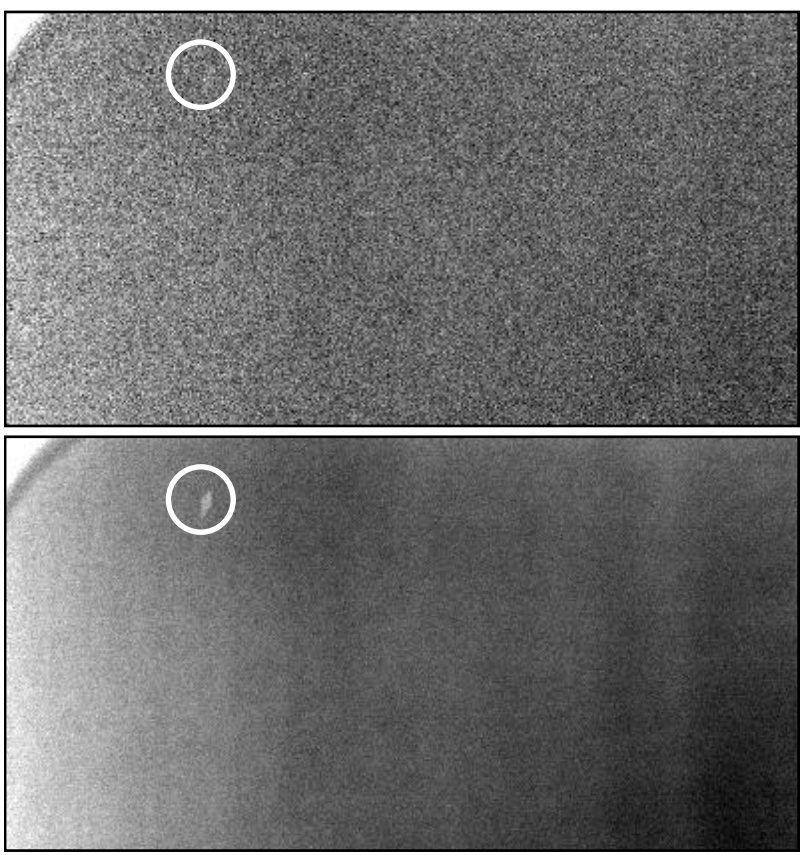

Figure 1. The effect of post process pixel binning on the quality of low dose images. The same phantom image is shown, with the same window/level, without (left) and with (right) pixel binning. It is easily recognised that the low contrast artefact (encircled) is more clearly visible on the binned image due to the decreased noise level.

ware (Image J, version 1.38 for Windows; National Institutes of Health, Bethesda, MD, USA). Different kernels $(2 \times 2,3 \times 3$, and $4 \times 4)$ were tested. All binned images were read by a senior screening radiologist and a mammography physicist. They compared the binned series with the original low dose image to subjectively determine the optimal binning settings.

\section{Results}

The range of acceptable lowest dose levels of W/Rh images, as indicated by three radiologists, differed per specimen and feature (Table 2). We observed that for presentation of normal breast tissue the quality was acceptable when the image was acquired with $10 \%-20 \%$ of the standard dose. Pathological masses could be presented acceptably at a $5 \%-20 \%$ dose level. For adequate depiction of clustered microcalcifications a $20 \%-40 \%$ dose fraction was required.

The $5 \%$ images acquired with a W/Ag target-filter combination at $32 \mathrm{kV}$ resulted in an average glandular dose (AGD) of $0.1 \mathrm{mGy}$. The diagnostic quality of the very low dose Ag-images was perceived as at least as good as the "diagnostically acceptable" image in the Rhseries. In two specimens (one with a stellate mass, one with microcalcifications) the Ag-images performed even better. They appeared to have the least loss of information, illustrated in Figure 2, with a more than reasonable di- 
Table 2. Fraction (\%) of AEC tube current with which the specific mammographic features (breast tissue (BT), mass (M) and microcalcifications (MCs)) were acceptably presented on a W/Rh image scored by 3 reviewers.

\begin{tabular}{|c|c|c|c|c|c|}
\hline & Specimen & Feature & R1 & R2 & R3 \\
\hline A & & $\begin{array}{c}\text { BT } \\
\text { M } \\
\text { MCs }\end{array}$ & $\begin{array}{l}15 \\
- \\
-\end{array}$ & $\begin{array}{c}10 \\
- \\
-\end{array}$ & $\begin{array}{c}15 \\
- \\
-\end{array}$ \\
\hline B & & $\begin{array}{c}\text { BT } \\
\text { M } \\
\text { MCs }\end{array}$ & $\begin{array}{c}20 \\
- \\
-\end{array}$ & $\begin{array}{l}15 \\
- \\
-\end{array}$ & $\begin{array}{c}20 \\
- \\
-\end{array}$ \\
\hline $\mathrm{C}$ & & $\begin{array}{c}\text { BT } \\
\text { M } \\
\text { MCs }\end{array}$ & $\begin{array}{l}20 \\
15 \\
-\end{array}$ & $\begin{array}{l}10 \\
5 \\
-\end{array}$ & $\begin{array}{c}15 \\
10 \\
-\end{array}$ \\
\hline D & & $\begin{array}{c}\text { BT } \\
\text { M } \\
\text { MCs }\end{array}$ & $\begin{array}{l}20 \\
20 \\
25\end{array}$ & $\begin{array}{l}15 \\
20 \\
40\end{array}$ & $\begin{array}{l}20 \\
20 \\
40\end{array}$ \\
\hline $\mathrm{E}$ & & $\begin{array}{c}\text { BT } \\
\text { M } \\
\text { MCs }\end{array}$ & $\begin{array}{c}15 \\
15 \\
-\end{array}$ & $\begin{array}{c}10 \\
20 \\
-\end{array}$ & $\begin{array}{c}15 \\
20 \\
-\end{array}$ \\
\hline$F$ & & $\begin{array}{c}\mathrm{BT} \\
\mathrm{M} \\
\mathrm{MCs}\end{array}$ & $\begin{array}{c}15 \\
- \\
25\end{array}$ & $\begin{array}{c}20 \\
- \\
20\end{array}$ & $\begin{array}{c}20 \\
- \\
25\end{array}$ \\
\hline $\mathrm{G}$ & & $\begin{array}{c}\text { BT } \\
\text { M (not shown) } \\
\text { MCs }\end{array}$ & $\begin{array}{l}20 \\
10 \\
25\end{array}$ & $\begin{array}{c}15 \\
5 \\
25\end{array}$ & $\begin{array}{l}20 \\
15 \\
40\end{array}$ \\
\hline
\end{tabular}

agnostic quality overall, even concerning microcalcifications (Figure 3). Optimal noise reduction was achieved by post process pixel binning with a $3 \times 3 \mathrm{kernel}$, resulting in improvement of perceived image quality and interpretability by expert opinion.

\section{Discussion}

Although the risk of radiation-induced breast cancer due to (screening) mammography is small $[7,8]$, minimising radiation exposure should always be pursued, particularly as the vast majority of the population involved is relatively young and asymptomatic.

This pilot study illustrates the potential of low dose images in mammography. Using a W/Ag target-filter combination for obtaining these images generates an image quality that is much better compared to $\mathrm{W} / \mathrm{Rh}$ imaging,
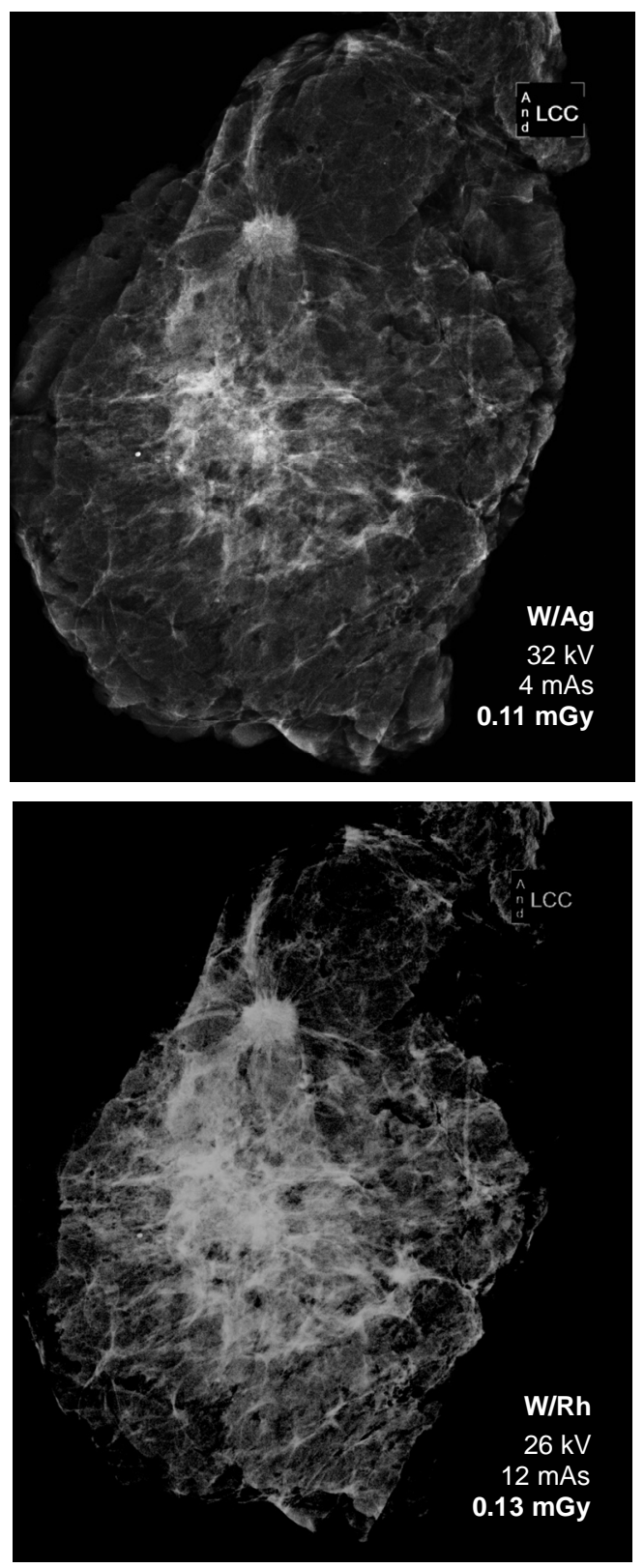

Figure 2. Difference in image quality using W/Ag or W/Rh in specimen imaging with a similar AGD and same window/ level settings.

as selected by the AEC, at similar dose level. While in standard practice a W/Ag spectrum is advised to be used for thicker breasts only [9], it shows advantages in low dose imaging irrespective of breast thickness, due to the more penetrating beams that compensate for the relative small amount of $X$-ray quanta. In this experimental setting the AGD could be reduced by approximately $78 \%-90 \%$, depending on breast composition and thickness. This suggests there is room for investigation of substantial dose reduction in clinical mammography images, without compromising considerably on diagnostic efficacy.

This result differs from previous study findings that 

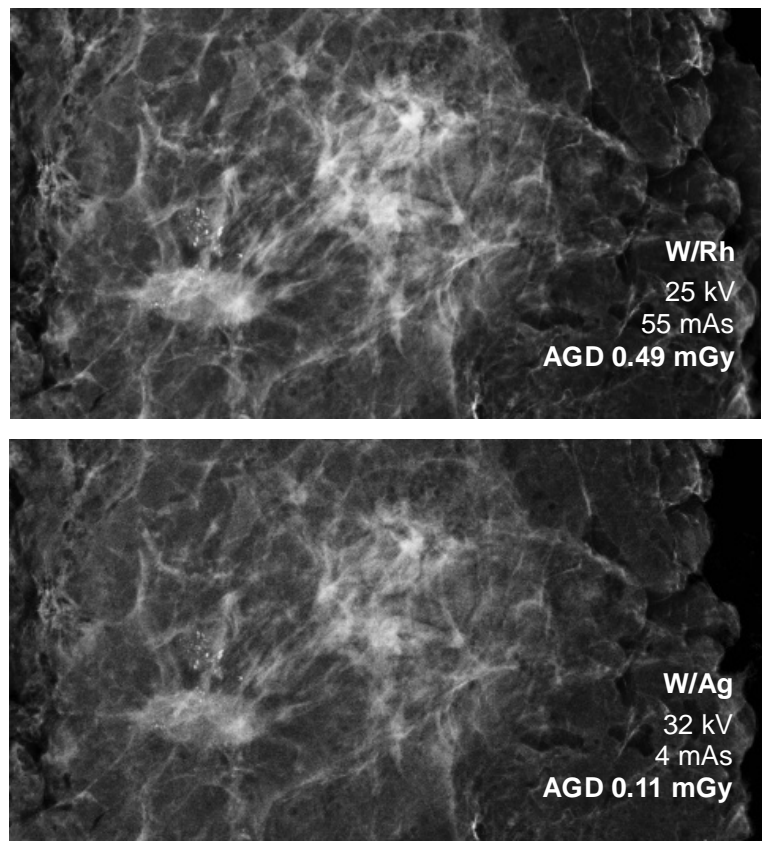

Figure 3. Standard AEC versus W/Ag low-dose specimen image with microcalcifications (same window/level).

conservatively suggested a $50 \%$ dose reduction in mammography [1-3]. One factor might be the use of mastectomy specimens here as study substrate. A phantom, as used by Samei et al. [1], does not have the ligaments and glandular structures of a breast, thereby lacking the most prominent source of noise, i.e. anatomical noise [10]. Processing real mammograms, as performed in the study by Chawla et al. [2], has the drawback of noise level and pathology simulation, which might be an oversimplified reflection of image modifications seen at low doses. But more importantly, our study had a different purpose. Previous studies were focussed on generalised dose reduction in mammography, with consequent concerns about the potential loss of image quality and the resultant effect on diagnostic efficacy. In this study, we were looking for a threshold radiation dose level for single views primarily, in a way they can add to standard mammography views, for example in the screening programme.

When using a $\mathrm{W} / \mathrm{Rh}$ target-filter combination we found that a higher dose level is needed for adequate depiction of clustered microcalcifications, compared to pathological densities. This finding is in accordance with previous observer performance study results $[1,11]$. However, with the use of a higher energy X-ray spectrum obtained with a $\mathrm{W} / \mathrm{Ag}$ combination, depiction of microcalcifications improved, due to the increase in detector dose level and associated lower quantum noise level.

Pixel binning improves the interpretability of the images by reducing uncorrelated noise; a technique that has already been applied in processing low dose images of breast tomosynthesis systems [12]. It has specific poten- tial in low dose imaging, as for low dose images the inevitable effect of reduced signal transfer at high frequencies, impairing detail analysis, is relatively small.

As the increased noise level in low dose imaging, especially in combination with noise reduction, might conceal subtle structures, such as microcalcifications, we believe substantial dose reduction should not be used in all mammography projections. However, these low dose images may be helpful as additional views, without a substantial increase in the absorbed average glandular dose. With standard images available for detection, additional low dose images can be useful for differentiation, for example to distinguish suspect lesions from summation artefacts (superimposition of normal structures). This might be true for screening mammography in particular, where recall rates are increasing after the introduction of digital mammography. As a consequence of the high contrast resolution detection increased, but pseudo lesions were often seen and recalled as well [13]. With additional projections this may be intercepted, thereby reducing false positive recalls [14]. Moreover, as an extra view, it is expected to contribute to the early detection of breast cancer $[15,16]$. Finally, low dose mammography images could be applied in post interventional mammography [17], such as marker or wire verification, or in high frequency surveillance.

This pilot study has some limitations that need to be addressed. First of all, the set of specimen is small, which makes it difficult to draw hard conclusions. Furthermore, it encompasses a subjective study. Nevertheless, it demonstrates the potential of low dose images obtained with certain parameters that gives us a tool for future research. The use of mastectomy specimens can be an advantage over other study substrates, but it will not be equivalent to mammography practice. Therefore, it is uncertain to what degree the results generalise; a shortcoming that is inherent to an experimental study set up.

\section{Conclusion}

In the digital era, low-dose mammographic views may have great potential in screening and diagnostic imaging practices. The observations of this pilot study suggest that application of a W/Ag spectrum for low dose imaging permits a substantial reduction of the AGD in single digital mammographic images, particularly when combined with post process noise reduction. These results encouraged us to start a clinical study focussed on the potential for dose reduction in specific mammography views as a feasibility test in mammography practice.

\section{REFERENCES}

[1] E. Samei, R. S. Saunders Jr., J. A. Baker and D. M. Delong, "Digital Mammography: Effects of a Reduced Ra- 
diation Dose on Diagnostic Performance," Radiology, Vol. 243, No. 2, 2007, pp. 396-404. doi:10.1148/radiol.2432061065

[2] A. S. Chawla, E. Samei, R. Saunders, C. Abbey and D. Delong, "Effect of Dose Reduction on the Detection of Mammographic Lesions: A Mathematical Observer Model Analysis," Medical Physics, Vol. 34, No. 8, 2007, pp. 33853398. doi:10.1118/1.2756607

[3] T. Svahn, B. Hemdal, M. Ruschin, D. P. Chakraborty, I. Andersson and A. Tingberg, "Dose Reduction and Its Influence on Diagnostic Accuracy and Radiation Risk in Digital Mammography: An Observer Performance Study Using an Anthropomorfic Breast Phantom," British Journal of Radiology, Vol. 80, No. 955, 2007, pp. 557-562. doi:10.1259/bjr/29933797

[4] D. R. Dance, A. K. Thilander, M. Sandborg, C. L. Skinner, I. A. Castellano and G. A. Carlsson, "Influence of Anode/ Filter Material and Tube Potential on Contrast, Signalto-Noise Ratio and Average Absorbed Dose in Mammography: A Monte Carlo Study," British Journal of Radiology, Vol. 73, No. 874, 2000, pp. 1056-1067.

[5] P. Bernhardt, T. Mertelmeier and M. Hoheisel, "X-Ray Spectrum Optimization of Full-Field Digital Mammography: Simulation and Phantom Study," Medical Physics, Vol. 33, No. 11, 2006, pp. 4337-4349. doi:10.1118/1.2351951

[6] E. L. Siegel and R. Khorasani, "To Compress or Not to Compress: A Compressed Debate," Journal of the American College of Radiology, Vol. 1, No. 12, 2004, pp. 981983. doi:10.1016/j.jacr.2004.09.014

[7] R. de Gelder, G. Draisma, E. A. Heijnsdijk and H. J. de Koning, "Population-Based Mammography Screening Below Age 50: Balancing Radiation-Induced vs Prevented Breast Cancer Deaths," British Journal of Cancer, Vol. 104, No. 7, 2011, pp. 1214-1220. doi:10.1038/bjc.2011.67

[8] M. J. Yaffe and J. G. Mainprize, "Risk of Radiation-Induced Breast Cancer from Mammographic Screening," Radiology, Vol. 258, No. 1, 2011, pp. 98-105. doi:10.1148/radiol.10100655

[9] P. Baldelli, N. Phelan and G. Egan, "Investigation of the Effect of Anode/Filter Materials on the Dose and Image Quality of a Digital Mammography System Based on an
Amorphous Selenium Flat Panel Detector," British Journal of Radiology, Vol. 83, No. 988, 2010, pp. 290-295. doi: $10.1259 / \mathrm{bjr} / 60404532$

[10] A. Burgess, "Human Observer Detection Experiments with Mammograms and Power-Law Noise," Medical Physics, Vol. 28, No. 4, 2001, pp. 419-437. doi:10.1118/1.1355308

[11] L. M. Warren, A. Mackenzie, J. Cooke, R. M. Given-Wilson, M. G. Wallis and D. P. Chakraborty, "Effect of Image Quality on Calcification Detection in Digital Mammography," Medical Physics, Vol. 39, No. 6, 2012, pp. 3202-3213. doi:10.1118/1.4718571

[12] D. Gur, G. S. Abrams, D. M. Chough, M. A. Ganott, C. M. Hakim and R. L. Perrin, "Digital Breast Tomosynthesis: Observer Performance Study," AJR American Journal of Roentgenology, Vol. 193, No. 2, 2009, pp. 586-591. doi:10.2214/AJR.08.2031

[13] A. M. Bluekens, N. Karssemeijer, D. Beijerinck, J. J. Deurenberg, R. E. van Engen and M. J. Broeders, "Consequences of Digital Mammography in Population-Based Breast Cancer Screening: Initial Changes and Long-Term Impact on Referral Rates," European Radiology, Vol. 20, No. 9, 2010, pp. 2067-2073. doi:10.1007/s00330-010-1786-7

[14] N. J. Wald, P. Murphy, P. Major, C. Parkes, J. Townsend and C. Frost, "UKCCCR Multicentre Randomised Controlled Trial of One and Two View Mammography in Breast Cancer Screening," BMJ, Vol. 311, No. 7014, 1995, pp. 1189-1193. doi:10.1136/bmj.311.7014.1189

[15] U. Bick and F. Diekmann, "What Do We and What Don't We Know?" European Radiology, Vol. 17, No. 8, 2007, pp. 1931-1942. doi:10.1007/s00330-007-0586-1

[16] R. G. Blanks, S. M. Moss and M. G. Wallis, "Use of Two View Mammography Compared with One View in the Detection of Small Invasive Cancers: Further Results from the National Health Service Breast Screening Programme," Journal of Medical Screen, Vol. 4, 1997, pp. 98101.

[17] C. C. Riedl, S. Jaromi, D. Floery, G. Pfarl, M. H. Fuchsjaeger and T. H. Helbich, "Potential of Dose Reduction after Marker Placement with Full-Field Digital Mammography," Invest Radiology, Vol. 40, No. 6, 2005, pp. 343-348. doi:10.1097/01.rli.0000163743.63063.9f 